\title{
Una modelación de los determinantes de la eficacia organizacional
}

\author{
Cruz García Lirios ${ }^{1}$
}

\begin{abstract}
RESUMEN
La discrepancia entre los valores humanos y las normas sociales son abordadas a partir de las aproximaciones institucionales (isomorfismo) y del comportamiento (toma de decisiones) organizacional para describir la lucidez (lo que se hace mediando él deber hacer y el poder hacer) como una variable que puede explicar lo que lleva a una organización pública a construir su campo de racionalidad, equilibrio, influencia y eficacia.
\end{abstract}

Palabras claves; valores humanos, eficiencia institucional, lucidez organizacional, y eficacia organizacional.

\section{INTRODUCCIÓN}

"Cuando tengas que elegir entre dos caminos, elige el más difícil" (proverbio del Tibet), esta frase puede definir lo que Saramago escribe en torno a la lucidez. La discrepancia entre dos mundos que han estado en conflicto cada vez que las organizaciones deben finiquitar problemáticas en las que las personas se preguntan ¿Quién ha firmado esto por mí?

Oceja y Jiménez (2001) proponen una clasificación normativa funcional. En principio, advierten que hay dos tipos de normas lo que debe hacerse y lo que se hace. Después, plantean que los determinantes de las normas pueden ser tres:

Probabilidad percibida de recibir una sanción formal cuando hay trasgresión de la norma (disuasión).

Presión que ejerce el grupo con respecto a dicha norma (influencia).

Grado de acuerdo que existe entre la norma y nuestros principios morales (legitimación).

Consecutivamente, proponen una tipología o conjunto estructurado y congruente de categorías que se derivan de combinar un conjunto de criterios. Los tipos de normas son:

Legitimas. Compatibles con nuestros principios personales.

Coercitivas. Incumplirlas implican sanciones aplicadas por una autoridad institucional con las que no sé esta de acuerdo.

Ilegitimas. Incumplirlas provoca sanciones formales con las que no estamos de acuerdo y no provocan desaprobación por parte de nuestro grupo de referencia.

\footnotetext{
${ }^{1}$ Universidad Autónoma Metropolitana. Correo electrónico: csh96327267@ titlani.uam.mx
} 
Prescriptivas. Compatibles con nuestros principios y se espera que el grupo de referencia las desapruebe en caso de incumplimiento.

Personales. Derivadas de nuestros principios y su incumplimiento no provoca sanción formal o informal alguna.

Reiteradas. Provocan una relación negativa con el grupo de referencia cuando son incumplidas y se percibe tanto una baja probabilidad de sanción como congruencia con nuestros principios.

Nulas. No están apoyadas por autoridades, nuestro grupo de referencia ni nuestros principios.

La aproximación institucional parece aplicar a pie juntillas esta clasificación de lo que debe hacerse versus lo que se hace.

Meyer y Rowan (1999) al definir reglas institucionales como “...clasificaciones incorporadas en la sociedad, como tipificadotas o interpretaciones intercambiadas.." (pag. 80), plantean el acceso de las normas sociales en la estructura burocrática de una organización. Dichas reglas institucionales (lo que las organizaciones deben hacer para legitimarse) determinarían su grado de institucionalización.

En este sentido, institucionalización puede indicar "...definir las funciones adecuadas para los negocios -como las ventas, la producción, la publicidad y contabilidad-..." (pag. 83)

Es decir, lo institucional es lo que debe hacerse para lograr una legitimidad (lo que será ovacionado al hacerse) complementaria con la eficacia (lo que se plantea llegar a lograr).

Cuando se ha logrado una optima institucionalización, puede transferirse de una organización a otra a través de mitos y ceremonias. Al resultado de este proceso, lo han llamado isomorfismo y lo han definido como "... proceso imitador que obliga a una unidad a parecerse a otras unidades que enfrentan las mismas condiciones ambientales..." (DiMaggio y Powell, 1999, pag. 108). Este proceso de similitud entre las organizaciones puede ser de tres tipos:

Coercitivo. Integrado por fuerzas políticas y su legitimidad impuesta.

Mimético. Sugiere un cambio de estructura globalmente reconocida y aceptada.

Normativo. Atribuible a la profesionalización (tradición y transferencia de un conocimiento)

Por su parte, la aproximación del comportamiento explicado a partir de la toma de decisiones, incluye la discrepancia entre lo ínter organizacional valido y lo intra organizacional necesario. 
Simón (1984) al describir los límites de la racionalidad, plantea que las decisiones se llevan a cabo a partir de una información incompleta que ni siquiera la imaginación puede sustituir. De esta forma, el proceso de racionalización se inicia con la Docilidad (ajuste del comportamiento con base en las consecuencias previstas para el logro de objetivos preestablecidos), pero no se consolida al no poder establecerse las alternativas de decisión y comportamientos implicadas en la decisión y consecuente conducta elegida.

De esta forma la organización sólo aspira a las siguientes dos fases.

Planificación esencial. Toma de decisión a partir de valores, métodos, conocimientos, habilidades e información.

Planificación de procedimiento. Diseño y establecimiento de los mecanismos que orientan la atención, canalizan la información y conocimientos.

En ambas, están implícitos cuatro mecanismos de Influencia Organizacional.

División del trabajo como la forma más ideal de llevarse a cabo.

Jerarquía de la toma de decisiones que soslaya las aportaciones individuales.

Intervención de los canales de información como la manipulación del comportamiento grupal a partir de los valores que les convenzan.

Adiestramiento como la adquisición de conocimientos y habilidades compartidas y llevadas a cabo en grupo.

En tal sentido, el Equilibrio Organizacional (disposición a participar y someterse a los objetivos de la organización) parece ser el siguiente nivel al que una organización llega después de racionalizarse e influirse a si misma (Simón, 1984). Los siete tipos de normas y los conceptos básicos de Isomorfismo, Influencia y Equilibrio Organizacional son indispensables para plantear la variable de Lucidez Organizacional la cual a continuación se describe y ejemplifica con las formulaciones de José Saramago (2004).

\section{DESARROLLO}

Tres indicadores de la Lucidez Organizacional pueden ser (1) las frases que la organización política (el primer ministro, los ministros del interior y cultura, así como sus empleados) comenta, (2) la estrategia que sigue la organización para culpar a la mujer que no quedo ciega y (3) la decisión de eliminar a las personas que amenazan su estatus de autoridad por falta de legitimidad (voto en blanco). Algunas frases implican la situación en la que se encuentra la organización una vez que inventa la relación causal entre la ceguera simulada de una mujer y el voto en blanco. Pareciera que se asume una postura sectaria ante un problema como el voto en blanco. En efecto, la secta es un elemento de la Lucidez Organizacional. 
Consecuentemente, la organización interviene los medios de comunicación para construir un complot que deslegitime el voto en blanco. De este modo, otro indicador de la Lucidez Organizacional es manipular los canales de comunicación. En el final del libro, la organización contrata a un asesino para eliminar al comisario y a la mujer que no quedo ciega. Esto indica que la Lucidez Organizacional es anticiparse a las consecuencias de las decisiones. También, la forma en como muere el comisario y después es irónicamente condecorado con el máximo reconocimiento del país, implica otro rasgo de la Lucidez organizacional: la martirización de sí misma.

Por lo tanto; la Lucidez Organizacional se origina a partir de un sectarismo que determina la manipulación de canales de comunicación en primera instancia, y la anticipación de estas consecuencias, en segunda instancia.

Ahora bien, para modelar las variables en un modelo, es indispensable la siguiente estrategia propuesta por Mithaug (2000) en cuatro fases:

1) Definición de un problema a partir de las discrepancias entre lo que se conoce y lo que se desconoce en torno a un evento o situación. En este sentido, se considera como principal discrepancia la aceptación de un comportamiento que al derivarse de un sistema económico y político Neoliberal y las necesidades organizacionales no puede ser un comportamiento eficaz.

2) Enfoque de la información perdida que puede explicar las discrepancias. Precisamente, la lucidez organizacional proporciona el contenido afectivo que las variables seleccionadas desde la aproximación institucional no han integrado.

3) Integración de la Información que cambie la condición desconocida hacia una conocida evaluando la credibilidad de la explicación construida y la validez, confiabilidad y utilidad de la nueva teoría. En tal sentido, se propone un modelo teórico con las variables seleccionadas.

4) Replicación de la estrategia de los cuatro pasos en la eliminación de otras inconsistencias entre la nueva teoría y las creencias existentes. Precisamente, se propone la contrastación empírica del modelo teórico.

Para derivar trayectorias causales de estas proposiciones, se plantea lo siguiente:

kerlinger y Lee (2002) definen el diseño de la investigación como “...el plan y la estructura de la investigación concebidos de manera que se puedan obtener respuestas a preguntas de investigación..." (pag. 317). De tal forma el plan de investigación es "...el esquema o programa general del estudio. Incluye un esbozo de lo que el investigador hará al escribir las hipótesis y sus implicaciones operacionales para el análisis final de los datos..." (pag. 317). En tanto la estructura es "...el marco conceptual, la organización o la configuración de elementos de estructura relacionados en formas específicas. La mejor manera de especificar una estructura consiste en escribir una ecuación matemática que relacione las partes de la estructura entre sí... es un paradigma o modelo de las relaciones entre las variables de un estudio..." (pag. 317). 
Respecto al plan y la estructura, Nunnally (1987) definen operacionalización como el error de medición en el que se incurre al sustituir un dato por una relación entre dos o más entidades y sólo se puede, por tanto, estimar un rasgo, una cantidad o evento de un atributo que este presente en un objeto.

De esta forma Cortés (1997) plantea que la causalidad indica juicios de probabilidad en torno a:

La variación concomitante (covarianza o correlación) entre variables y al efecto de una variable sobre otra.

Una asimetría temporal en la que se puede establecer una variable antecedente y otra consecuente.

La eliminación del efecto de otros constructos sobre los seleccionados a partir de la aleateorización de sus indicadores, comparación de muestras y regresión lineal.

De este modo, las variables se definen a partir de los siguientes tres criterios;

Se evita vaguedad, tautología y muchos indicadores.

Se establecen diferencias entre los indicadores de un constructo respecto a otros

Se establecen las condiciones específicas de su utilización

Por lo tanto, se considera que una variable es un conjunto de conceptos no observables en los cuales organismos, objetos, eventos o personas varían continuamente a partir de sus indicadores en un contexto de estudio dado y se modelan como exógenos, mediadores y endógenos. 
De este modo las variables tienen tres dimensiones a considerar (ver esquema 1).

Esquema 1. Dimensiones situacionales, espaciales y temporales de las problemáticas en torno al Decrecimiento y Crecimiento tanto Económico como Psicológico y el Desarrollo Sustentable en las organizaciones

\author{
Dimensión situacional \\ abundante de problemáticas
}

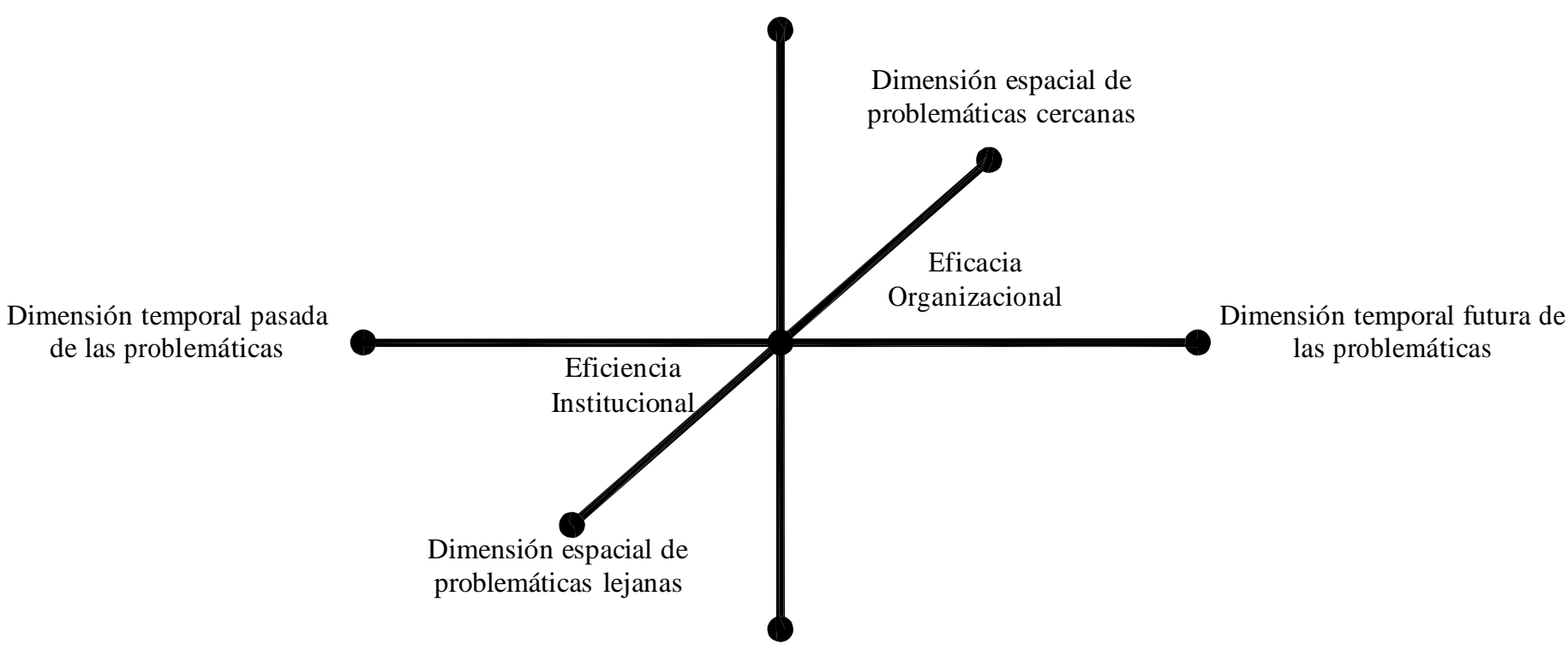

Dimensión situacional escasa de problemáticas

Fuente: Esquema elaborado a partir de la investigación de García-Mira y Guerrero (2000); García-Mira y Real (2001)

García-Mira y Guerrero (2000) demuestran con una muestra de 520 estudiantes de la Coruña España, mediante la técnica de escalamiento multidimensional (se asume que existe una relación lineal entre las proximidades y distancias euclidianas dentro del espacio de estímulos configurado por el conjunto de problemas ambientales mostrado a los individuos), dos categorías: global y local. La hipótesis demostrada plantea que la gente discrimina entre los ámbitos locales y globales en cuanto a la ubicación de los problemas ambientales. Es decir, la preocupación debe estar más orientada a los ítems de la categoría global. También realizaron el contraste entre los problemas de ahora y los que ocurrirán en 50 años. Concluyen que ambas dimensiones (local-global y persona-entorno) explican el $97.35 \%$ de la varianza total.

Por su parte, García-Mira y Real (2001a; 2001b) plantean que los acuerdos internacionales sobre la protección medioambiental sólo reflejan una hipermetropía generada por la lejanía 
de las problemáticas e información generalizada. Con una muestra de 126 trabajadores petroleros en Galicia España, demostraron mediante análisis de conglomerados y escalamiento multidimensional que la muestra discrimina entre los problemas globales y locales (69.77\%) así como en los ámbitos de implicación (13.08\%).

Respecto a la estructura de un modelo Kline (1998) la establece a partir de la formula:

Parámetros totales de un modelo $=$ parámetros de varianzas y covarianzas de los constructos exógenos + parámetros de varianzas y covarianzas de las perturbaciones de las trayectorias + parámetros de efectos directos e indirectos sobre los constructos endógenos. La tabla 1 muestra cuantos elementos deben incluirse en un modelo.

Tabla 1. Definición estructural

\begin{tabular}{|c|c|c|c|}
\hline Caso & Condiciones & Consecuencias & Soluciones \\
\hline $\begin{array}{l}\text { Infra } \\
\text { identificación } \\
\text { de parámetros }\end{array}$ & \begin{tabular}{|lr}
$\mathrm{Si}$ hay menos \\
incógnitas que valores \\
conocidos no se \\
pueden calcular las \\
ecuaciones & \\
estructurales & \\
\end{tabular} & $\begin{array}{l}\text { Proporción } \\
\text { observaciones } \\
\text { menor que } \\
\text { número } \\
\text { parámetros }\end{array}$ & $\begin{array}{l}\text { Disminuir las trayectorias } \\
\text { causales tanto de los } \\
\text { constructos exógenos comg } \\
\text { de los mediadores sobre el } \\
\text { endógeno a predecir }\end{array}$ \\
\hline $\begin{array}{l}\text { Plena } \\
\text { identifica } \\
\text { de parám }\end{array}$ & $\begin{array}{l}\text { Si hay igual número } \\
\text { de incógnitas con } \\
\text { valores conocidos se } \\
\text { pueden calcular las } \\
\text { ecuaciones } \\
\text { estructurales }\end{array}$ & $\begin{array}{l}\text { Proporción } \\
\text { observaciones igual } \\
\text { que el número de } \\
\text { parámetros }\end{array}$ & $\begin{array}{l}20 \text { observaciones (para } \\
\text { establecer el número de } \\
\text { observaciones considérese la } \\
\text { siguiente formula } \mathrm{n}(\mathrm{n}+1) / \\
2 \text { ) por cada parámetro } \\
\text { (proporción adecuada) }\end{array}$ \\
\hline $\begin{array}{l}\text { Sobre } \\
\text { identifica } \\
\text { de parám }\end{array}$ & $\begin{array}{l}\text { Si hay más incógnitas } \\
\text { que valores conocidos } \\
\text { no se pueden calcula } \\
\text { las ecuaciones } \\
\text { estructurales }\end{array}$ & $\begin{array}{l}\text { Proporción } \\
\text { observaciones } \\
\text { mayor que } \\
\text { número } \\
\text { parámetros }\end{array}$ & $\begin{array}{l}\text { Aumentar las trayectorias } \\
\text { causales tanto de los } \\
\text { constructos exógenos comg } \\
\text { de los mediadores sobre el } \\
\text { endógeno a predecir }\end{array}$ \\
\hline
\end{tabular}

Fuente: Tabla elaborada a partir del planteamiento de Kline (1998)

A partir de la diferencia entre una entidad moderadora y una mediadora. Baron y Kenny (1986) definen moderación como la fragmentación de los efectos directos de un grupo de dos o más entidades independientes sobre una entidad focal dependiente (ver esquema 2). Es decir, el efecto de una entidad independiente sobre una entidad focal dependiente disminuye o aumenta en función de la relación de una tercera entidad independiente con la primera entidad independiente. Dicho proceso moderador nos permite establecer cuándo ocurrirán los efectos expuestos. 
Esquema 2. Moderación: Fragmentación de los efectos directos de un grupo de entidades independientes sobre una entidad focal dependiente

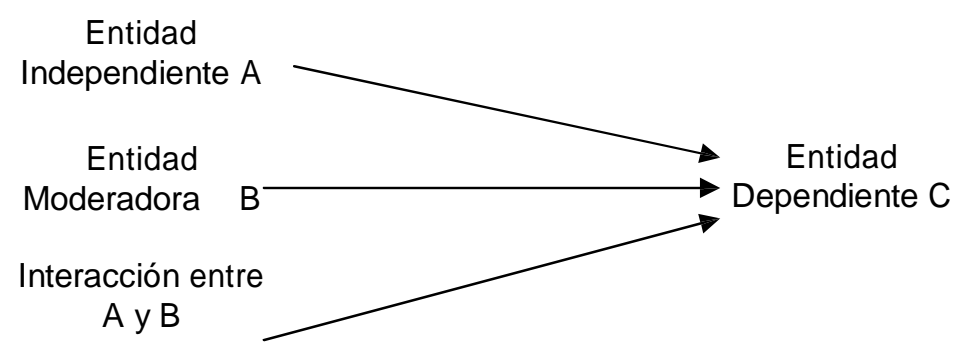

Fuente: Esquema elaborado a partir del planteamiento de Baron y Kenny (1986)

En el proceso moderador están incluidas tres condicionantes:

1) La entidad A debe estar directa y significativamente relacionada con la entidad $C$.

2) La entidad A no debe estar alta y significativamente relacionada con la entidad B.

3) La entidad B debe estar directa y significativamente relacionada con la entidad C.

En tanto, una mediación es la transmisión de los efectos de un grupo de entidades independientes sobre una entidad focal dependiente (ver esquema 3). Dicho proceso mediador devela cómo y por qué ocurren los efectos expuestos.

Esquema 3. Mediación: Transmisión de los efectos indirectos de un grupo de entidades independientes sobre una entidad focal dependiente a través de una entidad intermedia.

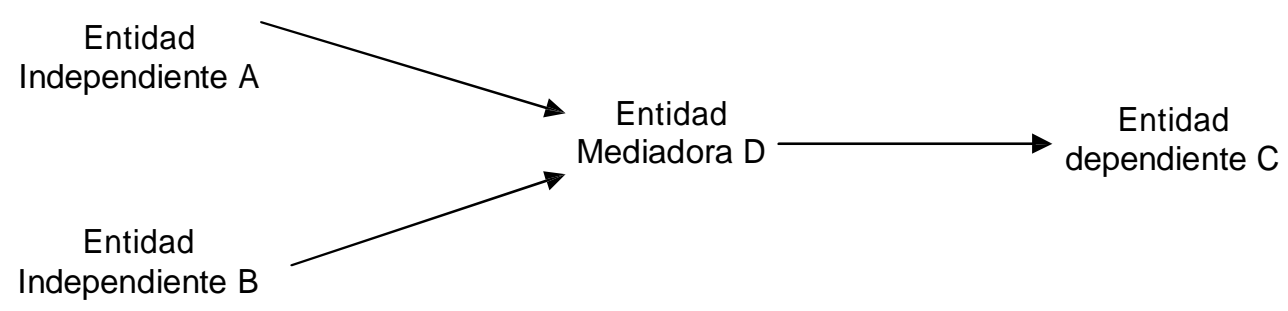

Fuente: Esquema elaborado a partir del planteamiento de Baron y Kenny (1986)

En el proceso mediador están incluidas tres condicionantes:

1) Las entidades A y B no deben estar directa y significativamente relacionadas con la entidad C.

2) La entidad $\mathrm{D}$ debe estar directa y significativamente relacionada con la entidad $\mathrm{C}$

3) Las entidades A y B deben estar alta y significativamente relacionados con la entidad D. 
Cabe señalar que los procesos moderadores son más frecuentes que los procesos mediadores. Por consiguiente, se exponen a continuación ambos procesos moderadores y mediadores en una notación estructural (ver esquema 4) .

\section{Esquema 4. Notación estructural}

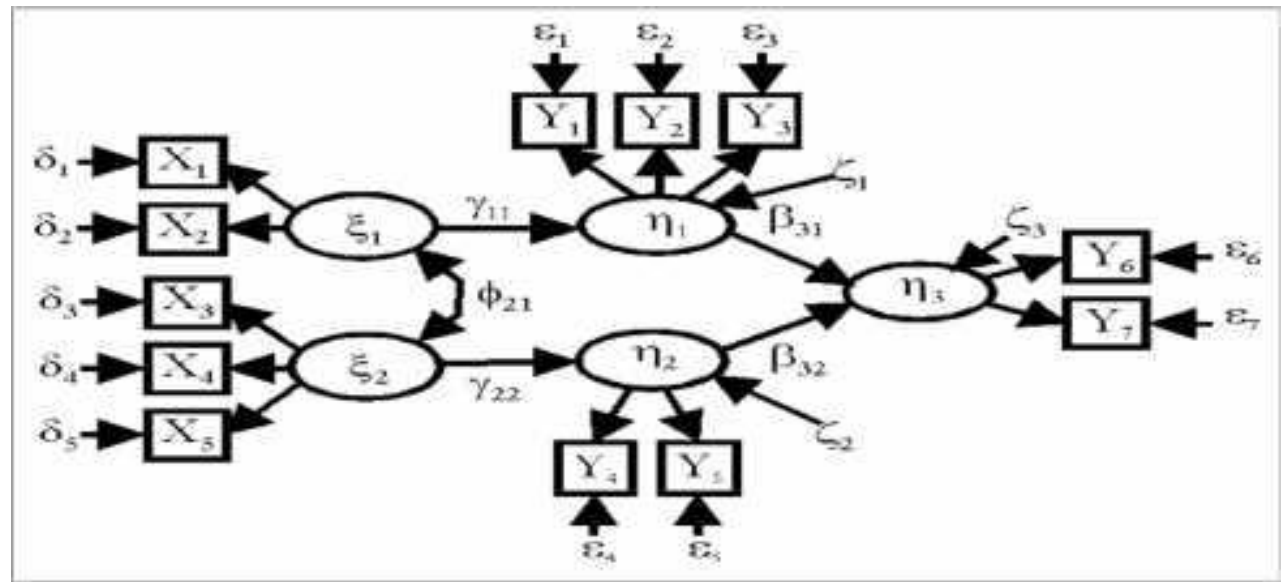

Fuente: Esquema elaborado a partir de una revisión de la literatura

$\xi(\mathrm{ksi})=$ Constructo exógeno porque delimita el inicio del modelo y los cálculos de las ecuaciones estructurales.

$\mathrm{X}=$ Indicadores del constructo exógeno

$\delta($ delta $)=$ Parámetro de disturbio (incidencia de otros constructos no incluidos en el modelo) de los indicadores del constructo exógeno

$\eta$ (eta) $=$ Constructo mediador y endógeno porque transmite y/o delimita el final de los efectos de los constructos exógenos.

$\mathrm{Y}=$ Indicador del constructo endógeno

$\varepsilon$ (epsilon) $=$ Parámetro del disturbio (incidencia de otros constructos no incluidos en el modelo) de los indicadores del constructo endógeno

$\beta$ (beta) = Parámetro de la regresión de un constructo mediador (transmite los efectos de constructos exógenos u otros endógenos) sobre otro constructo endógeno $\gamma($ gamma $)=$ Parámetro de la regresión de un constructo exógeno sobre un endógeno

$\phi($ phi $)=$ Parámetro de la covarianza entre constructos exógenos

$\zeta$ (zeta) $=$ Parámetro del disturbio (incidencia de otros constructos no incluidos en el modelo) estructural 


\section{CONCLUSIÓN}

El esquema 5 plantea la relación mediadora (transmite los efectos de otras variables) de la Lucidez (lo que se hace mediando el deber hacer y el poder hacer) con los Valores (lo que debe hacerse), con la Eficiencia (lo que puede hacerse decidiendo la mejor alternativa con base en una información limitada) y la Eficacia (lo que se espera llegar a hacer).

Esquema 5. Modelación de la discrepancia entre lo que debe hacerse y lo que puede hacerse
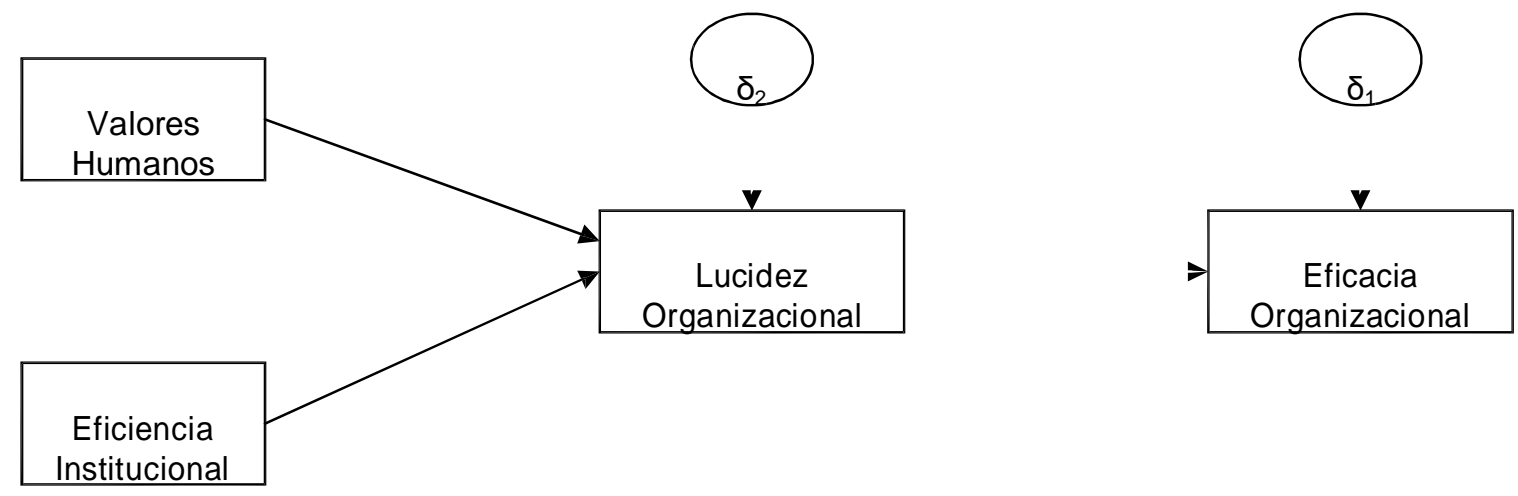

Finalmente, el modelo permitirá plantear el Crecimiento Organizacional no sólo reducido a lo económico, más bien integral. 


\section{REFERENCIAS}

Baron, R. M. \& Kenny, D. A. (1986). The moderator-mediator variable distinction in Social Psychology research: conceptual, strategic, and statistical considerations. Journal of Personality and Social Psychology. 51, 1173-1182.

DiMaggio, P. J. y Powell, W. W. (1999). Retorno a la jaula de hierro. El significado institucional y la racionalidad colectiva en los campos organizacionales. En W. W. Powell y P. J. DiMaggio (coords.). El nuevo institucionalismo en el análisis organizacional. (pp. 104-125) México: FCE-CNCPAP-UAM

García-Mira, R. y Guerrero, J. (2000). Problemas globales versus locales: dimensiones de preocupación ambiental. $1^{\circ}$ Congreso Hispano-Portugués de Psicología. Santiago de Compostela, España.

García-Mira, R. y Real, J. E. (2001a) Valores, actitudes y creencias: hacia un modelo predictivo del ambientalismo. Medio Ambiente y Comportamiento Humano. 2, 21-43.

García-Mira, R. y Real, J. E. (2001b). Dimensiones de preocupación ambiental: una aproximación a la hipermetropía ambiental. Estudios de Psicología. 22, 87-96.

Kerlinger, F. y Lee, H. (2002). Investigación del comportamiento. Métodos de investigación en Ciencias Sociales. México: McGraw Hill.

Kline, R. B., (1998). Principles and Practice of Structural Equation Modeling. New York: Guilford Press.

March, J. y Simon, H. A. (1977). Teoría de la organización. Barcelona: Ariel.

Meyer, J. W. y Rowan, B. (1999). Organizaciones institucionales: la estructura formal como mito y ceremonia. En W. W. Powell y P. J. DiMaggio (coords.). El nuevo institucionalismo en el análisis organizacional. (pp. 70-103) México: FCE-CNCPAPUAM

Nunnally, J. C. (1987). Teoría Psicométrica. México: Trillas.

Oceja, L. y Jiménez, I. (2001). Hacia una clasificación psicosocial de las normas. Estudios de Psicología. 22, 227-242.

Saramago, J. (2004). Ensayo sobre la lucidez. Madrid: Alfaguara.

Simon, H. A. (1984). El comportamiento administrativo: estudio de los procesos decisorios en la organización administrativa. Buenos Aires: Aguilar. 


\section{Anexos}

\section{Sinopsis}

José Saramago describe el dilema de servir a la organización o conducirse de acuerdo a ciertos principios éticos. Sucede que en una ciudad de Portugal, la gente ha asistido a votar pero no lo ha hecho por algún candidato de los tres partidos (derecha, centro e izquierda). Frente a este problema de legitimidad, las autoridades, organizan un atentado y después deciden poner en estado de sitio a la ciudad hasta que las personas reconozcan o denuncien a los autores intelectuales del voto en blanco. Durante muchos días las personas conviven minimizando las acciones represivas del estado. Mientras tanto, el primer Ministro junto con sus ministros de defensa, de cultura y del interior discute y planifican los mecanismos para que la gente cambie su actitud y acciones hacia el sistema democrático que el estado practica. Después de mucho tiempo, una carta llega a la oficina de un asistente del primer ministro, éste se asegura que no se difunda su contenido y ordena al ministro del interior que esclarezca su contenido, mismo que asigna el caso a un comisario y dos ayudantes. El comisario indaga la remota relación entre una mujer que no quedo ciega en la epidemia que azoto a la ciudad hace cuatro años y el voto en blanco, después de largas deliberaciones, trata de contrarrestar la propaganda del Estado hacia la mujer que no quedo ciega, culpándola de organizar el voto en blanco. El final de la novela es atroz, un asesino contratado por el Primer Ministro, ejecuta al comisario y a la mujer que no quedo ciega, cerrando con ello, una trama poco usual en las novelas políticas.

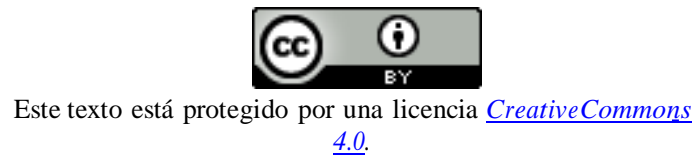

Usted es libre para Compartir - copiar y redistribuir el material en cualquier medio o formato - y Adaptar el documen- to - remezclar, transformar y crear a partir del material- para cualquier propósito, incluso comercialmente, siempre que cumpla la condición de:

Atribución: Usted debe reconocer el crédito de una obra de manera adecuada, proporcionar un enlace a la licencia, e in- dicar si se han realizado cambios. Puede hacerlo en cualquier forma razonable, pero no de forma tal que sugiera que tie-ne el apoyo del licenciante o lo recibe por el uso que hace. 